\title{
Turning points of recovery from cardiac surgery during the intensive care unit transition
}

\author{
Fu-Jin Shih, RN, DNSc, Shu-Hsun Chu, MD, Po-Jui Yu, RN, MSN, Wen-Yu Hu, RN, MSN, and \\ Guey-Shiun Huang, RN, MSN, Taipei, Taiwan, Republic of China
}

OBJECTIVE: To explore the turning points of recovery from cardiac surgery of patients during the intensive care unit recovery transition.

DESIGN: A descriptive qualitative design.

SETTING: Three hospitals in northern Taiwan, Republic of China.

PATIENTS: A convenience sample consisting of 30 adult patients (15 men and 15 women) who had undergone cardiac surgery. Age range was 20 to 67 years (mean, 46.6 years).

OUTCOME MEASURES: The component, context, type, and positive or negative impact of turning points on patients' health.

INTERVENTION: Data were collected through semistructured interviews and then analyzed by using qualitative content analysis.

RESULTS: Turning points, which included events, nursing actions, and time, were examined in terms of the preceding conditions and the positive or negative health outcomes. Participants in this study fell into four main groups with regard to turning point experiences during the intensive care unit transition: those having experiences of turning points with both positive and negative outcomes (57\%); those having experiences of turning points with a positive outcome only $(33 \%)$; those having no experience of a turning point with either a positive or negative outcome $(7 \%)$; and those having experiences of turning points with a negative outcome only (3\%). Four classes of turning point experiences identified from the data encompassed "precedingness," inclusiveness, comparativeness, and multiplicity.

CONCLUSIONS: We delineated the components, context, types, and positive or negative impact of turning points on patients' health after cardiac surgery during the intensive care unit transition. We also drew information on the nature, definition, and conceptual frameworks from data analysis to describe this phenomenon. (Heart Lung ${ }^{\circledR} 1997 ; 26: 99-108$ )

$\mathrm{R}$ ecovery from cardiac surgery during the immediate postoperative period in the intensive care unit (ICU) has been an issue of considerable interest to health care providers in the West. The stresses to which critically ill

\footnotetext{
From the College of Medicine, School of Nursing, National Taiwan University, and the Nursing Department, National Taiwan University Hospital, Taipei, Taiwan, Republic of China.

Supported by the National Science Council, Taiwan, Republic of China.

Reprint requests: Fu-Jin Shih, DNSc, RN, No. I, Section 1, Jen-Ai Road, Taipei, Taiwan, Republic of China 10018.

Copyright 01997 by Mosby-Year Book, Inc $0147-9563 / 97 / \$ 5.00+0 \quad \mathbf{2} / \mathbf{1 / 7 9 2 6 7}$
}

patients are exposed have been recognized since the inception of ICUs. Nearly three decades ago, researchers reported that $30 \%$ to $70 \%$ of ICU patients showed signs of high psychologic stress. ${ }^{1}$ Investigations have found that patients' perceptions in the ICU after cardiac surgery generally fall into five main categories: (1) physical discomfort, (2) psychologic responses, (3) cognitive responses, (4) sensory overstimulation, and (5) difficulty maintaining daily activities, such as sleeping patterns. $^{2-11}$

Patients' postoperative psychologic responses to cardiac surgery in the ICU include anxiety, depression, restlessness, irritability, panic, and anger. ${ }^{4-10}$ Their cognitive responses typically 
involve feelings of powerlessness, lack of control, and reduced self-esteem, such as with their body image. $4,5,10,11$ However, the impact on the recovery experiences of patients as a result of changes in these responses during the most critical stage is a matter that has not been adequately explored. Therefore, the following question arises: Is there any particular thing that patients who have under gone cardiac surgery view as their recovery marker (i.e., turning point or milestone), a particular thing that suddenly facilitates or hinders their recovery from cardiac surgery during the ICU transition? If the answer is yes, then it is vital to discover how these patients define such markers, what changes these markers cause, and what these changes signify to the patient who experiences them.

With knowledge of the markers (turning points) of recovery, health professionals may be able to help facilitate the recovery of patients from cardiac surgery during the most critical stage. The purpose of this study was threefold: first, to determine whether such turning points of recovery from cardiac surgery existed; second, to identify the components and nature of these turning points if they did exist; and last, to define the turning points of recovery from cardiac surgery.

\section{METHODS}

A qualitative research design was used because it is based on an inductive approach, which allows important dimensions of analysis to emerge from patterns found in the cases under study without first presupposing what these dimensions will be. ${ }^{12}$

Sample. The study protocol was reviewed and approved by the Committee for Research on Human Subjects at the University of California, San Francisco. Permission to conduct the study was also obtained from the directors of the Surgical and Nursing Departments of three general hospitals in northern Taiwan, each institution having a reputation for successful cardiac surgery. Because coronary artery bypass grafting ( $C A B G$ ), valvular replacement surgery (VRS), and atrial or ventricular septal defect repair are three major types of cardiac surgery in Taiwan, a convenience sample of subjects who met the following criteria was obtained: older than 18 years of age; able to understand and speak Mandarin, Taiwanese, or Hakka, or having family members willing to interpret for them; had undergone surgery for CABG, VRS, or atrial or ventricular septal defect repair; and had stayed in the ICU for at least 24 hours. Excluded were subjects who had had nasal-oral or tracheostomal tubes in place; were not fully conscious (Glasgow Coma Scale score $<15$ ); and had a degree of mental illness (confirmed by at least one psychiatric physician) before conducting interviews with the investigator.

Procedures. The list of all patients scheduled to receive cardiac surgery was obtained through daily contact with the clerk or head nurse of the surgical cardiovascular floor units at three hospitals. All patients who met the inclusion criteria and who expressed an interest in participating in the study before surgery were considered as potential candidates. They were then individually approached by the principal investigator, who informed them about the purpose of the study, the extent of participation expected, and the potential risks involved. A Chinese consent form was read to them before they were asked to participate. All patients were given time to read the consent form and ask questions. A signed Chinese consent form was obtained from each patient who chose to participate, and each patient was offered a copy of his or her consent form.

Instruments. Two types of data collection tools were used: a semistructured interview guide and the patient profile. The semistructured interview guide, with the title "Suggested Interview Questions and Probes for the Patient," was developed on the basis of literature reviewed pertaining to surgical cardiac recovery, as well as on Chinese health and culture. In addition, the principal investigator's empirical knowledge of more than 16 years of surgical cardiovascular ICU work in Taiwan and periodic (once or twice per week) consultation with three internationally respected interdisciplinary experts-a surgical cardiac nursing scientist, a nursing theorist, and a sociologist familiar with the use of multiple qualitative methods-also constituted valuable sources of input in devising this guide. The final modification of the interview guide was made after interviewing three male and two female Taiwanese patients who had undergone cardiac surgery in Taiwan before this study protocol was approved. The interviews were conducted in the patients' rooms on the surgical cardiovascular floor unit at least 24 hours after they left the ICU, when they felt comfortable to talk. The exact time of the interview was scheduled by consulting with the patient's primary nurse and obtaining the patient's agreement. Following the semistructured interview format, patients were asked questions with the aim of constructing a patient profile. The patient profile consisted of demographic information, such as the patient's age, language, marital status, socioeconomic status, living arrangements, 
and religious affiliation. After finishing the interview, the principal investigator recorded addition al demographic information from the patient's chart at the nursing station, including signs, symptoms, medical history, and medications.

Fifty-seven patients were recruited before the surgery, but only 30 of them $(15$ men and 15 women) actually met the requirements and completed the interview. Fifteen patients were too weak to participate or finish the interview because of fatigue or various postoperative complications, such as pulmonary edema, upper gastrointestinal tract bleeding, and cerebral vascular accident. Nine patients' family members insisted that patients should talk as little as possible to save energy for recovery from cardiac surgery, although the patients themselves had already agreed to participate in the study. The remaining three patients died during surgery or in the ICU.

Qualitative analytic methods. For the purpose of this study, the turning point of recovery from cardiac surgery was tentatively defined as "anything in particular (e.g., day, time, event) that made the patient suddenly feel much better or more comfortable regarding his or her health condition," "anything in particular that made the patient's mood suddenly become much worse regarding his or her health condition," "anything in particular that made the patient suddenly have more confidence in his or her health condition," and "anything in particular that made the patient suddenly lack confidence in his or her health condition."

Qualitative data first were transcribed from audiotape in the patient's native language (Mandarin, Taiwanese, or Hakka) with use of written Chinese and later were translated into English by the investigator and three individuals, all of whom were proficient in both Chinese and English, having a good knowledge of Eastern and Western culture, and well trained by the principal investigator. The accuracy of the data translation was checked by translating the English back into Chinese, and then having it reviewed by four other investigators. Data analysis took a total of 21 months, starting with the first interview and continuing to the end of the research period. To keep the emerging codes, categories, themes, and concepts firmly grounded in the subjects' actual experiences, a unique mode of qualitative content analysis was used. ${ }^{13,14}$

The various analytic codes, categories, and themes related to the concept of recovery expectations were developed by means of seven different levels of analysis. We developed this analytic approach by once or twice a week discussing the data with the aforementioned consultation group during the data analysis. The seven levels included (1) accuracy of transcription and translation; (2) obtaining a holistic understanding of the subjects' responses with research questions; (3) codifying emerging patterns in each interview and in field notes; (4) creating a work sheet to examine action and interaction strategy; (5) using the work sheet to examine action and interaction strategy; (6) coming up with themes by using data linkage and constant comparison; and (7) generating categories and subcategories.

Other strategies were also used to enhance the credibility of the findings. Informant checking was agreed to by all respondents. ${ }^{15-17}$ Negative cases were investigated and analyzed. ${ }^{17-19}$ All patients' charts were reviewed. ${ }^{16,19}$ In addition, each subject's primary nurse or caregiver was asked to confirm specific events. ${ }^{15,17,20}$

\section{RESULTS}

Sample demographics. The participants ranged in age from 20 to 67 years (mean, 46.6; SD, 15.3 years); 15 were men and 15 were women. The majority of the participants were married. Most had received more than 10 years of schooling. Ninety percent were employed. Twenty-three percent had an annual family income of between $\$ 22,641$ and $\$ 36,226$. For these subjects, their lower range was used as their income figure. Because two of the subjects had irregular income, the mean income of the other 28 subjects $(\$ 30,136)$ was used for purposes of analysis. More than $50 \%$ considered their annual family income adequate. The severity of cardiac disease ranged from New York Heart Association (NYHA) functional class I $(43 \%)$ to III $(27 \%)$. Thirty percent of subjects had CABG; others had septal replacement surgery, or VRS, or both CABG and VRS, or other surgeries.

The duration of stay in the ICU ranged from 1 to 20 days (mean, 5.3; SD, 3.3 days). The majority $(83 \%)$ stayed in the ICU for 1 week or less, $13 \%$ for 8 to 14 days, and $3 \%$ for more than 2 weeks. The period of time after leaving the ICU at which subjects felt comfortable to give the interview ranged from 1 to 13 days (mean, 5.4; SD, 2.4 days) (Table).

Patient experience with turning points of recovery. Taiwanese patients described several types of experiences regarding turning points (markers). Fifty-seven percent of the subjects $(n=17)$ had experiences of turning points with both positive and negative outcomes. Thirty-three percent $(n=10)$ had experiences of turning points with a positive outcome only. Seven percent $(n=2)$ had no experience of a turning point with either a positive or 


\section{Table}

Sample demographics $(n=30)$

\begin{tabular}{lccrr} 
Demographics & Mean (SD) & Range & \multicolumn{1}{c}{$\boldsymbol{n}$} & \% \\
\hline Age $(\mathrm{yr})$ & $46.60(15.34)$ & $20-30$ & 5 & 17 \\
& & $31-40$ & 6 & 20 \\
& & $41-50$ & 5 & 17 \\
Education & & $51-60$ & 7 & 23 \\
\end{tabular}

Graduate school

College

Senior high school

Junior high school

Elementary school

27

None

Occupation

Not working

Housewife

Self-employed

Laborer

Administrator

Teacher

Student

Family income/ $y \mathrm{r}^{*}$

(NT $26.5=$ US $\$ 1$ )

NT 533,642 (288,874)

NT 40,000-99,999

NT 100,000-199,999

NT 200,000-299,999

NT 300,000-399,999

NT 400,000-499,999

NT 500,000-600,000

$>\mathrm{NT} 600,000$

Perceived adequacy of income

Very satisfied

Satisfied

Okay

Unsatisfied

Very unsatisfied

Number of caregivers

$2.03(0.96)$

NYHA functional class

I

II

III

$2.03(0.96)$

600,000

1
2
3
4

Type of surgery

CABG

VR

SR

Redo VR

CABG and VR

SR and annuloplasty

Annuloplasty

No. of days in SCVICU

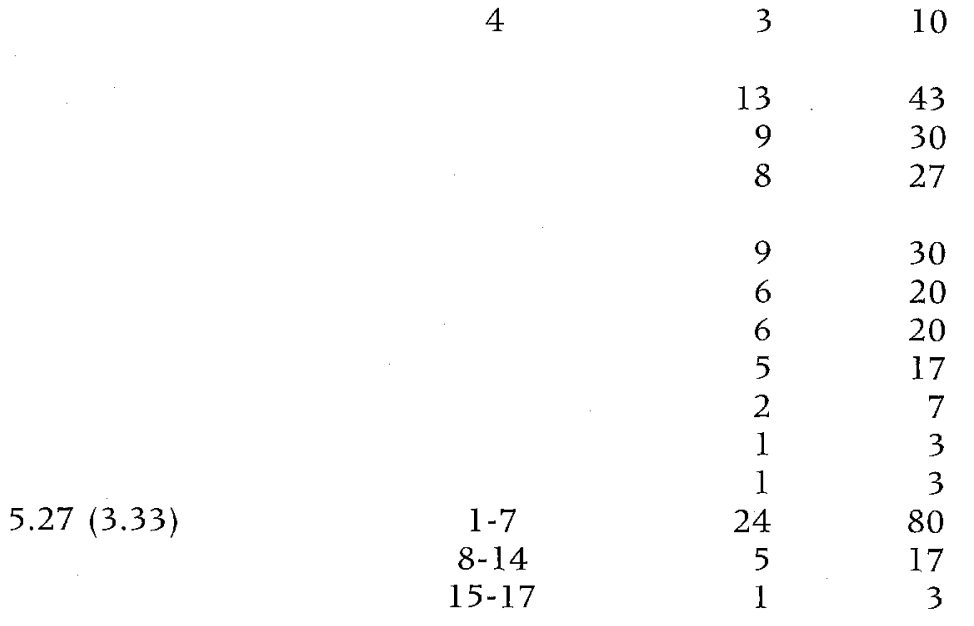

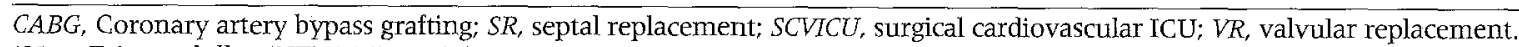
*New Taiwan dollar (NT) $26.5=$ US $\$ 1$. 
negative outcome, and $3 \%$ of the subjects $(n=1)$ had experiences of turning points with a negative outcome only.

Context for turning points with positive outcomes. Six preceding conditions were found to provide a context for perceived markers with positive outcomes during the ICU recovery transition. These conditions are (1) having concerns about mortality ("I stayed in the ICU for 30 days since I had an asthma attack; sometimes I thought I would die in the ICU"), being uncertain of recovery ("I didn't know how well I had recovered"), and not being sure about when they will be able to leave the ICU; (2) lacking full consciousness ("I was not fully conscious until that day [turning point]"); (3) having to endure physiologic pain from endotracheal or nasogastric tube, and having other discomforts, such as dry mouth, obstruction of airways, and inability to cough up sputum ("the [endotracheal and nasogastric] tubes rubbed against my throat, and it caused irritation and pain in my throat"; "I couldn't cough the sputum out until that time [turning point]"); (4) feeling nervous or under stress ("before the surgery, of course, I was very nervous"; "all stress was relieved after the surgery [turning point occurred]"); (5) perceiving abnormality or not feeling fully recovered because the surgical incisions had not healed ("my incisions had not healed until that day [turning pointl") or being unable to eat, move, or talk before extubation ("I couldn't move before that time Iturning pointl"; "I was in too much pain to talk [before removing endotracheal tube]"); and (6) perceiving being slighted by health professionals, such as physicians or nurses ("I felt I was being slighted until they came to see me [turning point occurred]").

Types of turning points with positive outcomes. Ninety-three percent of the subjects described three types of turning points with some positive outcomes in their ICU period. Of the turning points with a positive outcome, most were events $(87 \%)$, some were nursing actions $(17 \%)$, and a few were times $(10 \%)$.

Events that were perceived as markers with positive outcomes included the occasion when they first saw family members after the operation; when they first opened their eyes in the ICU or first became conscious; when they started to eat orally; when they were first visited by health professionals; when "I felt my condition got better" or when they were told they had recovered; when they were informed that they would be able to leave the ICU, or when they were transferred from the ICU to the floor unit; and when they received a certain treatment, such as the removal of their tubes, including the endotracheal tube, nasogastric tube, chest tubes, and urinary catheter.
Nursing actions that were defined as markers with positive outcomes included providing fastacting pain medication (such as giving injections to patients with postoperative pain), providing oxygen mask therapy, feeding, vibrating the back, and monitoring the intravenous drip.

The subjects who identified "time" as a marker with positive outcomes described how certain postoperative days were perceived as being significant to how they felt. For one, it was the fourth postoperative day, when her physical pain first started to subside. For another, it was the eleventh postoperative day, when he resumed full consciousness. For two, it was the day they were informed that they would be able to leave the ICU, or the day they were transferred from the ICU to the floor unit. One patient thought it was a period of time (duration), such as "after the surgery" (since "all stress was relieved after the surgery").

Positive impact of turning points on patients' health. Subjects gave further details about how the markers (events, nursing actions, and times) had a positive impact on their health. The consequences of these turning points included a feeling of having survived ("I thought, 'Finally the surgery is over; I've come back, like I had a long sleep"'; "now I've come back to the world of reality"); resuming full consciousness ("I was not fully conscious until the eleventh postoperative day"; "when I first became conscious, I didn't know whether I'd been through surgery or not, until the nurse fed me; then I realized that maybe I'd gone through surgery"); suddenly becoming Chin-Son (Mandarin, meaning physically or psychologically relaxed or both) ("I was so Chin-Son after I heard the doctor's decision to let me leave the ICU"; "I didn't have any pain from that day on; I felt so Chin-Son"; "I felt more Chin-Son the next day, because I saw nurses handling my IV"); perceiving normality or recovery ("I felt I had recovered at the moment when I was transferred from the ICU to the floor unit"); perceiving being respected by health professionals ("they lhealth professionals] didn't look down upon me"); being able to be accompanied by family members ("when I could see my husband's face, I was satisfied"); expecting to leave the ICU soon ("I felt that I was going to leave the ICU soon; leaving the ICU was my greatest wish"); being freed from worry ("all stress was relieved after the surgery"; "I no longer worried any more at that time"); and having good moods ("I felt most happy at that moment").

Context for turning points with negative outcomes. Sixty percent of the subjects $(n=18)$ experienced turning points with some negative outcome. Fifty-seven percent $(n=17)$ had experi- 
ences of a turning point with both positive and negative outcomes, and only $3 \%(n=1)$ had experiences of a turning point with a negative outcome only. Subjects also described six preceding conditions as being contexts for perceived markers or turning points with negative outcomes during their ICU period. These conditions were (1) having no concern about the surgery ("I didn't worry about anything before the surgery [turning-point]"); (2) not suffering or not experiencing much Tong-Kou (Mandarin, meaning physiologic or psychologic pain or both) caused by dyspnea ("I started to have breathing problems after that time [turningpoint]; I felt so Tong-Kou"), tracheal bleeding, spasms, bruises to the body ("my body had so many bruises, I felt so Tong-Kou"), sensations of nausea, having sore bones or skin, or enduring intense physical pain ("I had never been in so much pain before"); (3) having no difficulty or only slight difficulty in maintaining daily activities, such as sleep or moving ("I slept well before the surgery Iturning-pointl"; "I still could go to the restroom by myself before the surgery [turning-point]"; (4) having good moods ("my moods were pretty good before they treated me in that way Iturning-point occurredl"), and perceiving no vulnerability ("I didn't feel I was such a poor thing until they treated me like that (turning-point occurred]"); (5) having the will to survive ("after that [turning-point occurred], I really didn't want to live"); and (6) having no trouble with the nurses ("I had always followed her orders up until that moment [turningpointl"), and having only one endotracheal tube placed ("they [nurses] tried to insert another tube into my mouth").

Types of turning points with negative outcomes. Just over one half of the subjects $(53 \%)$ described certain events as turning points in their recovery. Nearly a third $(30 \%)$ described nursing actions as turning points not supportive of recovery. Thirteen percent found timing as a turning point associated with negative outcomes during their ICU period.

Events that were perceived as markers with negative outcomes included having shortness of breath, severe coughing, being unable to fall asleep, suffering physiologic pain or discomfort or both, vomiting, the IV line being disconnected, and having treatments, such as extubation and reintubation.

Subjects also described how they perceived certain postoperative dates or moments to be negative signs of recovery and significant to how they felt. Some subjects thought the first night in the ICU was the particular time during which they felt most Tong-Kou, because they had regained consciousness, yet were unable to fall asleep and could not talk to the nurses as a result of intubation. Others considered the second day in the ICU to be such a time, because they had undergone extubation and perceived it as a painful process.

Nursing actions that were defined as markers with negative outcomes included drawing arterial blood frequently and only after multiple attempts, suctioning, verbally threatening the patient, and labeling the patient as uncooperative.

Negative impact of turning points on patients' health. Negative consequences of these turning points were (1) having difficulty in maintaining daily activities; for example, not getting enough sleep because of pain, being unable to move freely as a result of both hands being restrained ("I couldn't do anything, except to turn myself a little bit, since my hands were tied up"), or feeling fatigue ("It made me feel so tired, since I couldn't fall asleep"); (2) suffering physical discomfort, such as shortness of breath ("I was coughing so much that it was hard to breathe"), vomiting ("the tube in my nose was inserted into my throat, so I always felt nauseated; I always wanted to throw up"), sore bones and skin ("I lay there and it felt like my skin and bones were all becoming fragile"), intense Tong-Kou as a result of being unable to fall asleep ("I felt very Tong-Kou because when I wanted to sleep, I couldn't"), massive tracheal bleeding, purposive or accidental extubation, reintubation, suctioning sputum, postoperative pain, spasms, or getting bruises ("I got bruises all over my body; I felt so much pain, I mean Tong-Kou"); (3) suffering psychologic Tong-Kou caused by fighting with nurses, because subjects tried to remove their tubes themselves ("the moment when I felt the most Tong-Kou was when she Ithe nursel said, 'If you shout again, I'll punch you in the mouth!'; she was so mean!"; "I exerted myself to try to spit the tube out with my teeth; I made an effort to push the tube out of my mouth"); (4) having concerns, worries, or fears ("I thought I was already short of blood, and now I had lost so much more blood"; "I felt so scared"); (5) perceiving vulnerability ("That night I considered myself to be such a poor thing, and that night seemed like it was a month long") and having bad moods ("my mood became very bad Isince she had to comply with the nurses' orders|"); (6) perceiving a poor recovery ("I felt my health condition was getting worse"); and (7) losing the will to survive ("I really didn't want to live any longer").

No turning point with negative outcome experiences. Some $40 \%$ of the subjects did not have any 


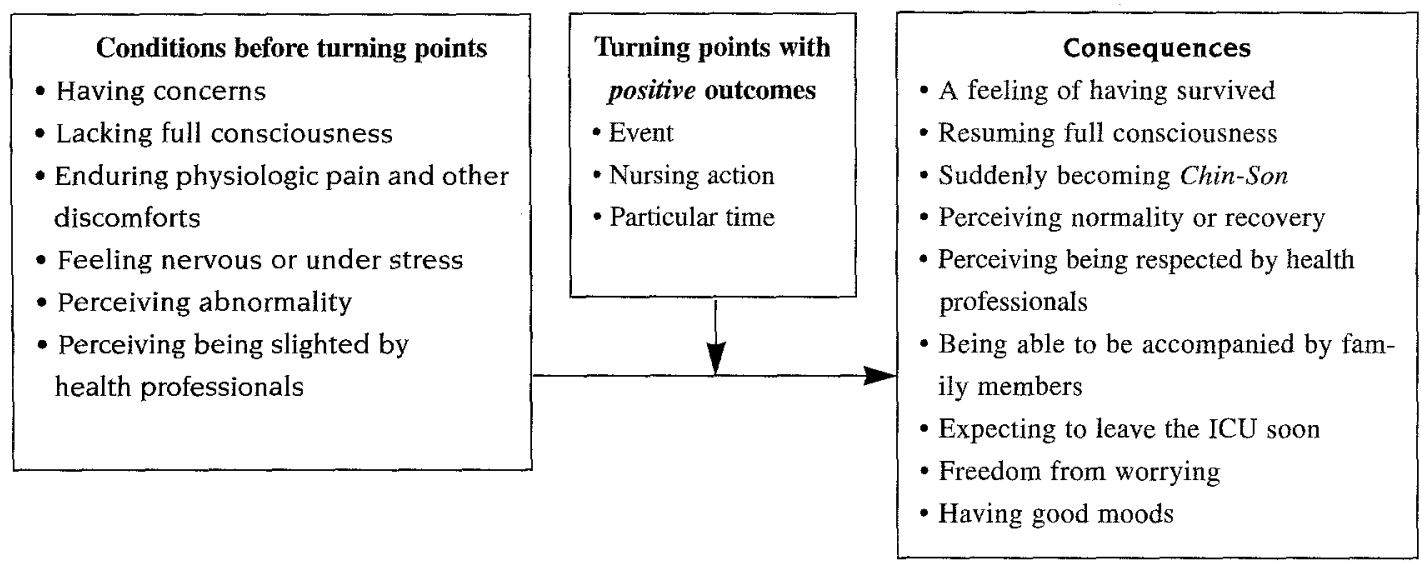

Fig. 1 Conceptual framework of Taiwanese patients' perceptions of tuming points with positive outcomes during ICU transition.

\begin{tabular}{|c|c|c|}
\hline $\begin{array}{l}\text { Conditions before turning points } \\
\text { - Having no concern } \\
\text { - Not suffering, or not experiencing } \\
\text { much Tong-Kou } \\
\text { - Having no difficulty, or only slight dif- } \\
\text { ficulty, in maintaining daily activities } \\
\text { - Having good moods and perceiving no } \\
\text { vulnerability } \\
\text { - Having the will to survive } \\
\text { - Having no trouble with the nurses and } \\
\text { undergoing intubation with only one } \\
\text { endotracheal tube }\end{array}$ & $\begin{array}{l}\text { Turning points with } \\
\text { negative outcomes } \\
\text { - Event } \\
\text { - Nursing action } \\
\text { - Particular time }\end{array}$ & $\begin{array}{l}\text { Consequences } \\
\text { - Having difficulty in maintaining daily } \\
\text { activities } \\
\text { - Suffering physical discomfort or Tong- } \\
\text { Kou } \\
\text { - Suffering psychologic Tong-Kou } \\
\text { - Having concerns, worries, or fears } \\
\text { - Perceiving vulnerability and having } \\
\text { bad moods } \\
\text { - Perceiving a poor recovery } \\
\text { - Losing the will to survive }\end{array}$ \\
\hline
\end{tabular}

Fig. 2 Conceptual framework of Taiwanese patients' perceptions of turning points with negative outcomes during ICU transition.

experience of a turning point with a negative outcome, although some of them experienced turning points with a positive outcome. Several reasons were offered by the patients as explanations. Some believed they had survived the surgery and recovered smoothly because they had complied with health professionals' orders. For example, "I need to follow their orders. I don't move without their orders. I don't try to remove the tubes by myself. Then, I'll be okay." Others did not worry about their recovery because they trusted the physician's skills and considered the medical facilities in the ICU to be "excellent." Still others attributed their lack of worry about recovering to their self-confidence. Finally, some cited the importance of psychologic support. For example, "the nurses and my family members always gave me a lot of An-Wei (Mandarin, meaning comfort), so I didn't have that experience."
No turning point experiences. Two subjects (7\%) were unable to articulate any experience of a turning point during their stay in the ICU. They had either prepared themselves for surgery or consid ered themselves to have recovered smoothly during the ICU period.

\section{DISCUSSION}

This study shows that turning point experiences were prevalent in Taiwanese patients during the ICU transition, with $93 \%$ of the subjects $(n=28$ ) reporting such experiences. The main findings to emerge from this study consisted of deeper insights into the nature of this phenomenon and a greater ability to define and theoretically describe it.

components and classes of turning points of recovery. The turning point experiences of Taiwanese patients followed a sequence of three components: context (preceding conditions), 
markers, and consequences. Four classes of turning point experiences were identified from the data. The first one is "precedingness," which refers to cases where the conditions before markers occurred seemed to provide a baseline for the particular patient to decide whether the specific event, nursing action, or time led to positive or negative health consequences. Most of the preceding conditions for markers with positive outcomes-having concerns, lacking full consciousness, enduring physiologic pain or discomfort, feeling nervous or stressed, and perceiving abnormality-were similar to the responses of Western patients in the ICU after cardiac surgery, $4,7,8,10$ except for the perception of being slighted by health professionals; which was expressed by some elderly subjects in this study. In addition, some elderly female patients mentioned being threatened or labeled as uncooperative by the nurses as markers with negative outcomes. In contrast to this, a trusting relationship with health professionals and receiving psychologic support from them were seen by some subjects as important reasons why they did not experience turning points with a negative outcome. Subjects in this study seemed to expect and require care with more respect and psychologic support. This observation may be related to the patients' social and cultural background, because in Chinese culture health professionals are expected to treat their patients as if they were relatives. ${ }^{5,21-23}$ Therefore, Taiwanese patients usually expect health care providers (and nurses in particular) to be their comforters by demonstrating the attitude of Chin Cha (meaning polite, courteous, and respectful) and providing care with great patience. ${ }^{5}$

The second class of recovery turning points is inclusiveness, which refers to those cases in which turning point experiences were not mutually exclu sive, because one person might experience several turning points with both positive and negative health outcomes.

The third class of turning point experience is comparativeness, which indicates situations where the consequences caused by the marker are related to preceding conditions. Thus, the preceding condition and its consequences need to be examined together rather than separately. The conclusion that emerges from the data in these cases is that the consequences of a marker would not exist without certain preceding conditions, and these consequences are generally opposite in nature to the conditions that preceded the change. However, to determine the overall trajectory of the change, as well as to understand clearly the connection between preceding conditions, markers, and consequences, further investigation is required.

The fourth class of turning point experience is multiplicity, which refers to cases involving multiple markers, dimensions, and levels. One person may experience multiple turning points, and their consequent changes may affect multiple dimensions. Patients' turning point experiences may encompass more than one change in their health condition. The changes (consequences) caused by a turning point may be physical, cognitive, or psychosocial in nature or may be a holistic perception. Examples from this study of changes in the physical dimension include suffering physical discomfort or Tong-Kou, having difficulty in maintaining daily activities, and resuming full consciousness. Examples of cognitive changes include perceiving abnormality or normality, expecting to leave the ICU soon, and losing or gaining the will to survive. Psychosocial changes encountered in this study include suffering from psychologic Tong-Kou, having bad or good moods, having concerns or freedom from worrying, perceiving vulnerability, being slighted or respected by health professionals, and being accompanied by family members. The holistic perceptions recorded include a feeling of "survival" and "suddenly becoming Chin-Son." In Chinese culture, Chin-Son is a feeling that embraces and transcends all of these dimensions. Nevertheless, this unusual state of being calls for further investigation, because little literature documents it. Moreover, patients' turning point experiences were not limited to the intrapersonal level but also involved interpersonal relationships, such as interactions with family members and relationships with health professionals.

Conceptual definition and conceptual frameworks of turning points. The initial definition of turning point used in this study may now be tentatively modified to become "something (event, nursing action, or particular time) that suddenly affects the patient's preceding condition and leads to a more positive or negative outcome with regard to their health." The conceptual frameworks drawn from data analysis depicting patients' turning point experiences are presented in Figures 1 and 2 .

Finally, although the definition, type, components, and classes of turning points of recovery from cardiac surgery were revealed by analysis of the data, the precise nature of the relationship between patients' perceived degree of recovery and their experiences of turning points is still unknown. Moreover, the link between factors that often have a tremendous impact on surgical cardiac recovery, such as status of the surgery (elective, 
urgent or emergent, primary versus redo), severity of the disease (in particular, the ventricular function of the patient), age, and sex, and patients' experiences of turning points was not documented either. Future researchers are encouraged to consider examining the aforementioned relationships with a larger sample size of varying socioeconomic levels. The definition, type, and nature of turning points of recovery from cardiac surgery are all elements requiring continuous examination and comparison over time. In addition, determining reliable turning point indicators and carefully recording the turning points seen during different stages of recovery are issues that deserve attention. Finally, the conceptual frameworks developed in this study also need further validation, modification, and development.

Though a need remains for ongoing research in this area, several practical suggestions based on the results of this study can be made. First, our findings may sensitize critical care nurses to detect more quickly and evaluate more precisely the turning point experiences of patients. Critical care nurses may apply the findings in this study to facilitate the recovery of their patients by creating or maintaining the preceding conditions of markers leading to positive outcomes, reinforcing markers having positive outcomes and their consequences, and being more sensitive to identify and prevent markers associated with negative outcomes or at least to minimize their effects if the conditions are unpreventable.

Second, nursing educators can incorporate the findings of this study into surgical cardiovascular nursing education programs for nursing clinicians and students to help them better understand the turning point experiences of patients during the ICU transition. Nursing diagnosis, nursing care plans, and strategies aimed at exploring turning points of recovery from cardiac surgery may also be further developed.

Third, principles derived from the cases of subjects who did not have turning point experiences with negative outcomes may also serve as valuable preoperative and postoperative guidelines for critical care nursing clinicians. Health care providers are encouraged to create and maintain a trusting relationship with their patients by respecting their feelings and needs and providing psychologic support. It is vital as well that the patient's need for quality care and medical treatment during the most critical recovery stage be adequately met.

Finally, nurses on the floor units may educate patients before surgery on the basis of knowledge of the turning points of recovery. If this teaching is done, the patients will better understand their recovery process and will be better prepared for cardiac surgery.

Several limitations are inherent in this study. The first concerns the reliability of the interview data, because the time for the interviews was not standardized. The subjects' perceptions of their turning points were based on recollections anywhere from 1 to 13 days after leaving the ICU, depending on when they felt comfortable enough for the interview.

Another limitation lies in the representativeness of the sample. Subjects with multiple types of cardiac surgery were recruited to increase the diversity of the sample and enhance the richness of the data for the purpose of strengthening the adequacy and credibility of this qualitative work. However, this approach might make it less advisable to generalize the findings to a specific cardiac population. In addition, because most subjects were from the middle or upper class and had a NYHA functional class rating of less than IV, the findings in this study are only directly applicable to patients with the aforementioned characteristics, rather than being valid for all patients undergoing cardiac surgery. The experience of turning points of recovery may differ for patients who undergo nonelective cardiac surgery, have poor ventricular function, come from a lower socioeconomic class, or lack a primary caregiver(s).

The third limitation is related to the validity of the data. The process of transcription, followed by translation from Chinese into English and the carrying out of data analysis based on the English version, may have resulted in some loss of meaning and accuracy.

\section{CONCLUSIONS}

Using a qualitative research design based on data from the patient's perspective, we explored the phenomenon of turning points of recovery from cardiac surgery in a sample of Taiwanese patients. We examined the types, components, classes, definition, and conceptual framework of turning points. More than $90 \%$ of the participants experiences that are "something (event, nursing action, or particular time) which suddenly affects the patient's preceding condition and leads to a more positive or negative outcome with regards to their health" during the ICU transition. From the standpoint of the health outcome, four groups of turning point experiences were identified: those with both positive and negative outcomes, those with positive outcomes only, those with negative outcomes only, and those with no turning points. The components of turning points we identified 
were preceding conditions, markers, and consequences. The classes of turning points that emerged from the data analysis were precedingness, inclusiveness, comparativeness, and multiplicity. With knowledge of turning points (markers), experiences of recovery from the patients' perspectives, the discrepancies between the patients' and critical care nurses' perceptions of recovery from cardiac surgery may be decreased. The critical care nurses would also be empowered to provide more culturally competent care for the patients during their most critical recovery transition from cardiac surgery.

This project was made possible with the help of Drs. Afaf I. Meleis, Susan R. Gortner, Virginia G. Oleson, and Strauss L. Anselm.

\section{REFERENCES}

1. DeMeyer J. The environment of the intensive care unit. Nurs Forum 1967;6:263-72

2. Baker CF. Sensory overload and noise in the ICU: sources of environmental stress. Crit Care Nurs Q 1984;6:66-80.

3. Haslam P. Noise in hospitals: its effect on the patient. Nurs Clin North Am 1970;5:715-24

4. Riegel B. Stressors of critically ill patients. In: Riegel B, Ehrenreich D, editors. Psychological aspects of critical care. Rockville (MD): Aspen, 1989:17-30.

5. Shih Fl. The experiences of Taiwanese patients during recovery transitions from cardiac surgery [dissertation]. San Francisco: University of California San Francisco. Ann Arbor (MI): UMI, 1995; University Microfilms No. 9502643:93-290.

6. Vanson RI, Katz BM, Krekeler K. Stress effects on patients in critical care units from procedures performed on others. Heart Lung 1980;9:494-7.

7. Wilson VS. Identification of stressors related to patients' psychological responses to the surgical intensive care unit. Heart Lung 1987;16:267-73.
8. Blacher RS, Cleveland RI. Paradoxical depression after heart surgery. In: Speidel H, Rodewald G, editors. Psychiatric and neurological dysfunctions after open heart surgery. New York Thieme, 1980:141-3.

9. Hwang SL, Lin BG, Liou MG, Chang E, Hwang SF. Stressors associated with heart surgery - a follow up and comparison study of patients' and nurses' perceptions of pre- and postheart surgery stressors. NAROC Nurs Research, 1994;2:17-27.

10. Kern LS, Norris SO, Constancia P. Coronary artery bypass grafting. In: Riegel B, Ehrenreich D, editors. Psychological aspects of critical care. Rockville (MD): Aspen, 1989;150-70

11. Wright JE. Self-perception alterations with coronary artery bypass surgery. Heart Lung 1987;16:483-90.

12. Patton $\mathrm{MO}$. Qualitative evaluation method. Rockville (MA): Newbury Park (CA): Sage, 1980:44-5; 306-18

13. Manen MV. Researching lived experience. New York: State University of New York Press, 1990.

14. Morgan DL. Qualitative content analysis: a guide to paths not taken. Qual Health Res 1993;3:112-21.

15. Emerson R, Pollner M. On the use of members' respondents to researchers' accounts. Human Organ 1983;47:189-98.

16. Hammersley M, Atkinson P. Ethnography principles in practice. New York: Routledge, 1990:195-200.

17. Woods NF, Catanzaro M. Nursing research theory and practice. St. Louis: Mosby, 1988.

18. Strauss A, Corbin I. Basics of qualitative researchGrounded theory procedures and techniques. Newbury Park (CA): Sage, 1990

19. Katz I. Contemporary field research-a collection of readings Emerson RM, editor. Prospect Heights. (IL): Waveland 1983:127-48,

20. Fielding NG, Fielding JL. Linking data: the articulation of qualitative and quantitative methods in social research. Beverly Hills (CA): Sage, 1986:41-53.

21. Chen-Louie T. Nursing care of Chinese American patients. In Orque MS, Bloch B, Monrroy LSA, editors. Ethnic nursing care: a multicultural approach. St. Louis: Mosby, 1983:183 218 .

22. Shih FJ. Perception of self in the intensive care unit after cardiac surgery among adult Taiwanese and American-Chinese patients. Int J Nurs Stud 1997;34:17-26.

23. Shih FJ. Concepts related to Chinese patients' perceptions of health, illness and person: issues of conceptual clarity. Accid Emerg Nurs 1996;4:208 15

\section{BOUND VOLUMES AVAILABLE TO SUBSCRIBERS}

Bound volumes of Heart \& Lung ${ }^{\circledR}$ are available to subscribers (only) for the 1997 issues from the Publisher at a cost of $\$ 30.00$ for domestic, $\$ 38.52$ for Canadian, and $\$ 36.00$ for international subscribers for Vol. 26 (January-December). Shipping charges are included. Each bound volume contains a subject and an author index and all advertising is removed. Copies are shipped within 60 days after publication of the last issue in the volume. The binding is durable buckram with the Journal name, volume number, and year stamped in gold on the spine. Payment must accompany all orders. Contact Mosby-Year Book, Inc., Subscription Services, 11830 Westline Industrial Dr., St. Louis, MO 63146-3318, USA; phone (800)453-4351 or (314)453-4351.

Subscriptions must be in force to qualify. Bound volumes are not available in place of regular Journal subscription. 